\title{
Clinically significant genomic alterations in the Chinese and Western patients with intrahepatic cholangiocarcinoma
}

Shifeng Xu' ${ }^{1 \dagger}$, Yuan Guo ${ }^{2 \dagger}$, Yanwu Zeng ${ }^{3}$, Zhijian Song ${ }^{3}$, Xiaodan Zhu ${ }^{4}$, Ning Fan ${ }^{5}$, Zhilei Zhang ${ }^{6}$, Guibing Ren ${ }^{7}$, Yunjin Zang ${ }^{8^{*}}$ (D) and Wei Rao ${ }^{8^{*}}$

\begin{abstract}
Background: The goal of this study is to disclose the clinically significant genomic alterations in the Chinese and Western patients with intrahepatic cholangiocarcinoma.

Methods: A total of 86 Chinese patients were enrolled in this study. A panel of 579 pan-cancer genes was sequenced for the qualified samples from these patients. Driver genes, actionability, and tumor mutational burden were inferred and compared to a cohort of Western patients.

Results: Totally, 36 and 12 driver genes were identified in the Chinese and Western cohorts, respectively. Of them, seven driver genes (IDH1, KRAS, TP53, BAP1, PBRM1, ARIDIA, and NRAS) were shared by the two cohorts. Four driver genes (SPTA1, ARID2, TP53, and GATA1) were found significantly correlated with the tumor mutational burden. For both cohorts, half of the patients had actionable mutations. The two cohorts shared the most actionable genes but differed much in their frequency. Though KRAS mutations were at the first and second actionable rank respectively for the Chinese and Western populations, they were still at a relatively low level of actionable evidence.
\end{abstract}

Conclusions: The study on the clinical significance of genomic alterations directs the future development of precision medicine for intrahepatic cholangiocarcinoma treatment.

Keywords: Intrahepatic cholangiocarcinoma, Genomic alteration, Population, Clinical significance, Driver gene, Actionability

\section{Background}

The Chinese population has a higher incidence rate of intrahepatic cholangiocarcinoma (iCCA) in comparison to the Western population [1]. However, the incidence rate in the Western population is now rapidly increasing. Patients with iCCA regularly have poor prognosis. In a cohort study [2], half of the patients who underwent curative resection had a 5-year survival rate at 19\% and

\footnotetext{
*Correspondence: zangyj3657@qq.com; qdfy_raowei@126.com

${ }^{+}$Shifeng Xu and Yuan Guo contributed equally to this work.

${ }^{8}$ Organ Transplant Center, The Affiliated Hospital of Qingdao University, Haier Road No. 59, Qingdao 266000, Laoshan District, China

Full list of author information is available at the end of the article
}

a median survival of 27.6 months. With the advent of precision medicine, targeted therapy and immunotherapy would essentially reshape the treatment of iCCA, especially for those unresectable patients.

To start targeted therapy or immunotherapy, genotyping is the first step. Zou et al. [3] reported a landscape of intrahepatic cholangiocarcinoma in the Chinese population by whole-exome sequencing. They revealed eight driver genes in 103 iCCA patients. Those genes were TP53, KRAS, IDH1, PTEN, ARID1A, EPPK1, ECE2, and $F Y N$. Another study in the Western population conducted by Lowery et al. [4] compared intrahepatic and extrahepatic cholangiocarcinomas with a panel of 410

(C) The Author(s). 2021 Open Access This article is licensed under a Creative Commons Attribution 4.0 International License, which permits use, sharing, adaptation, distribution and reproduction in any medium or format, as long as you give appropriate credit to the original author(s) and the source, provide a link to the Creative Commons licence, and indicate if changes were made. The images or other third party material in this article are included in the article's Creative Commons licence, unless indicated otherwise in a credit line to the material. If material is not included in the article's Creative Commons licence and your intended use is not permitted by statutory regulation or exceeds the permitted use, you will need to obtain permission directly from the copyright holder. To view a copy of this licence, visit http://creativecommons.org/licenses/by/4.0/. The Creative Commons Public Domain Dedication waiver (http://creativecommons.org/publicdomain/zero/1.0/) applies to the data made available in this article, unless otherwise stated in a credit line to the data. 
genes. By sorting the frequency of mutations in patients, they got eight commonly mutated genes in intrahepatic and extrahepatic cholangiocarcinoma including $I D H 1$, TP53, ARID1A, BAP1, KRAS, PBRM1, SMAD4 and $A T M$. Besides, there was also a 30 -patients study comparing different types of biliary tract cancer with a 22gene panel by Hogdall et al. [5]. They identified three significantly mutated genes including ARID1A, TP53, and $K R A S$ in CCA (cholangiocarcinoma). All the above studies concentrated on the mutations in one population, neglecting the difference between populations. In considering the significant incidence difference between the Chinese and Western populations, it would be interesting to know the clinical significance of their shared and distinct genomic alterations.

In this study, we collected 86 samples from Chinese iCCA patients. Since different researches used different methods to call the driver genes, directly comparing the results from multiple pieces of research could be problematic. Thus, we used the same pipeline to analyze driver genes, actionability, and tumor mutation burden and compared them between the Chinese and Western cohorts.

\section{Methods}

\section{Patient and sample preparation}

This study was approved by the ethical committee of the Affiliated Hospital of Qingdao University and Shandong Provincial Hospital Affiliated to Shandong First Medical University. A total of 86 Chinese patients with intrahepatic cholangiocarcinoma were enrolled (ORI dataset). Each participant had provided written informed consent. Samples were collected from surgery after diagnosis.

\section{Sequencing}

Sequencing was conducted in a CLIA/CAP-compliant Molecular Diagnostics Service laboratory of Origimed Co., Ltd. The detailed sequencing procedure was described as our previous work [6, 7]. Briefly, tissue samples were collected for each patient. KAPA Hyper Prep Kit (Illumina) was used to extract DNA. At least $50 \mathrm{ng}$ of double-stranded DNA was required for further library construction. Barcodes were added to the segments for multiplex sequencing to reduce the false discovering rate. From both segments' ends, 151 bp nucleotides were read on an Illumina Novaseq 6000 (Illumina, San Diego, CA). Two samples did not pass library quality control. Totally, 84 samples had been sequenced.

\section{Variation calling}

A total of 579 pan-cancer genes (Yuansuo ${ }^{\circ}$ from Origimed incorporation, Shanghai, China) were captured by targeted amplification. Variation calling methods were referred to that used by the MSK cohort (https:/github.com/rhshah/
IMPACT-Pipeline). Briefly, reads were mapped to the UCSC hg19 reference sequences using BWA MEM (version 0.7.9a) [8]. Redundant reads were de-duplicated. GATK [9] was used to recalibrate base quality. Variants were called by Mutect [10], Pindel [11] and Somatic Indel Detector [9]. Structural variants were identified by Delly [12].

\section{Driver gene identification}

To remove the false positive mutations or passenger mutations, the driver genes were identified by MutSigCV [13] using the GenePattern web service [14]. The prebuild full exome coverage file, covariate file and genome file for hg19 from GenePattern web service were used. The $p$-values for all the panel genes were inferred by MutSigCV. Significantly mutated genes were determined by the $p$-values adjusted by the Benjamini \& Hochberg method.

\section{Pathway analysis}

The enriched KEGG pathway [15] and gene ontology were analyzed using the DAVID web service [16]. The significant items were determined by the false discovery rate with a threshold at 0.05 .

Clinical actionability annotation of the somatic mutations Clinical actionable mutations were annotated with a python software MafAnnotator.py published by OncoKB [17]. The parameter, disease type, was set "CHOL". Mutations were annotated with six levels, they were, "Level_1", “Level_2A", "Level_2B", "Level_3A", "Level_ 3B" and "Level_4" [17].

\section{Tumor mutational burden analysis}

Tumor mutational burden (TMB) was calculated as the number of mutations multiplied by a adjust factor. The adjust factors were 1.1152 and 0.9738 for the two assays from the MSK dataset, and 0.7875 for the ORI dataset. The TMB difference between mutated and wild type patients was compared by the Mann-Whitney U test. The false discovery rate (FDR) was used to adjust the $p$ values.

\section{Public dataset}

A dataset from a Western cohort was prepared by Zehir et al. [18] and downloaded from cBioportal [19]. This cohort enrolled 87 patients from multiple ethnicities with intrahepatic cholangiocarcinoma in the United States by Memorial Sloan Kettering Cancer Center.

\section{Statistical test}

Fisher's exact test was used for the frequency comparison between the two cohorts. The Benjamini-Hochberg procedure was used for the correction of multiple 
testing. Power estimate of the two patient cohorts was performed with an R package "exact2x2" [20].

\section{Results}

The landscape of somatic mutations among the Chinese and Western populations

A total of 86 Chinese patients with primary intrahepatic cholangiocarcinoma were enrolled in this study (ORI dataset). The characteristics of these patients were listed in Table 1. The median age at diagnosis of the Chinese patients was 59, ranging from 18 to 83 years old. For comparison, we also included a cohort dataset (MSK) curated by Zehir et al. [18], which consisted of 87 iCCA patients from the Western population. The median age at diagnosis of the Western patients was 65 years old, ranging from 37 to 79 . The Chinese cohort had a younger age than the Western cohort at diagnosis $(p$-value $=$ 0.024, Mann-Whitney U test).

Samples from Chinese iCCA patients were sequenced by CLIA/CAP-compliant Molecular Diagnostics Service laboratory of Origimed Co., Ltd. Of the 86 samples, two samples did not pass the quality control and only the left 84 samples were sequenced. Figure 1 showed the top frequent mutations in the Chinese population and the Western population. The five top mutated genes in the Chinese population were TP53, KRAS, ARID1A, PBRM1, and SMAD4. The five top mutated genes in the Western population were IDH1, ARID1A, BAP1, TP53, and KRAS.

We also identified gene fusions in ORI with the same method as MSK cohorts. FGFR2 fusions are most identified in both populations. There were 27 FGFR2 fusion events in the ORI cohort and 33 in the MSK cohort. FGFR2 was most fused with WAC in the ORI cohort and BICC1 in the MSK cohort, respectively. Supplemental Table 2 lists all the fusion events in both populations.

Table 1 Clinical characteristics of patients with intrahepatic cholangiocarcinoma

\begin{tabular}{llll}
\hline Characteristics & & ORI & MSK \\
\hline Age & Median (range) & $59(18-83)$ & $65(37-79)$ \\
Sex & Male & 48 & 35 \\
& Female & 38 & 52 \\
Stage & I & 29 & 0 \\
& II & 8 & 0 \\
& III & 22 & 0 \\
Histological grade & LV & 3 & 58 \\
& Unknown & 24 & 29 \\
& Middle & 2 & 0 \\
& High & 37 & 0 \\
& Unknown & 29 & 0 \\
\end{tabular}

\section{Driver genes in both populations}

To reduce the possible false positive, we utilized MutSigCV [13] to identify the driver gene. The same full genome coverage file was used as a control for both populations. There were 36 and 12 significantly mutated genes identified for ORI and MSK datasets, respectively (Fig. 2a) (Supplemental Table 1). Among them, seven mutated genes (KRAS, TP53, BAP1, IDH1, PBRM1, $A R I D 1 A$, and NRAS) were shared. And, 29 and 5 driver genes were ORI-specific and MSK-specific, respectively. Most shared driver genes had a higher mutation allele frequency in both cohorts (Fig. 2b). Gene ontology analysis of the biological processes revealed enriched functions in macromolecule modification, regulation of cell proliferation, and positive regulation of metabolic process for the ORI cohort (Fig. 2c). MSK cohort was enriched in the glyoxylate cycle, regulation of neuron death and regulation of cell proliferation (Fig. 2d). KEGG pathway analysis identified significant pathways including melanoma, generic cancer and endometrial cancer in the ORI cohort (Supplemental Fig. 1) but none in the MSK cohort.

\section{Clinical actionability of genomic variations}

To reveal the treatment potential, mutations were classified into six levels of drug actionability evidence proposed by OncoKB [17]. Each mutation was assigned a level according to the highest actionability proof strength. Then we summarized the best treatment for each patient. For the ORI cohort, $60 \%$ of patients had actionable mutations (Fig. 3a). For the MSK cohort, $66.27 \%$ of patients had actionable mutations (Fig. 3b). Among them, 11.76 and $27.71 \%$ of patients had standard care biomarkers predictive of response to an FDAapproved drug (Level_2B). Next, we studied whether the mutations in each gene had an equal actionability for both populations. The actionability for each mutation was summarized for each gene. The highest actionable level was chosen for each gene in a patient. Then we counted the number of patients for each gene by the highest levels. ORI cohort had top actionable mutations in KRAS, CDKN2A, PIK3CA, and FGFR2 (Fig. 3c). MSK cohort had top actionable mutations in IDH1, KRAS, FGFR2, ATM, IDH2, and NRAS (Fig. 3d). Both cohorts had a similar set of actionable genes, differing only in their frequency (Fig. 3c and d).

\section{Mutation profile of typical genes}

Mutation distribution on the important driver genes could result in different clinical outcomes and treatment efficacy. BRAF was such an example in the ORI cohort. As shown in Fig. 4a, BRAF had two mutations (V600E and $\mathrm{K} 601 \mathrm{~N}$ ) in the patients of the ORI cohort with Level_2B and Level_3B actionability, respectively. BRAF 


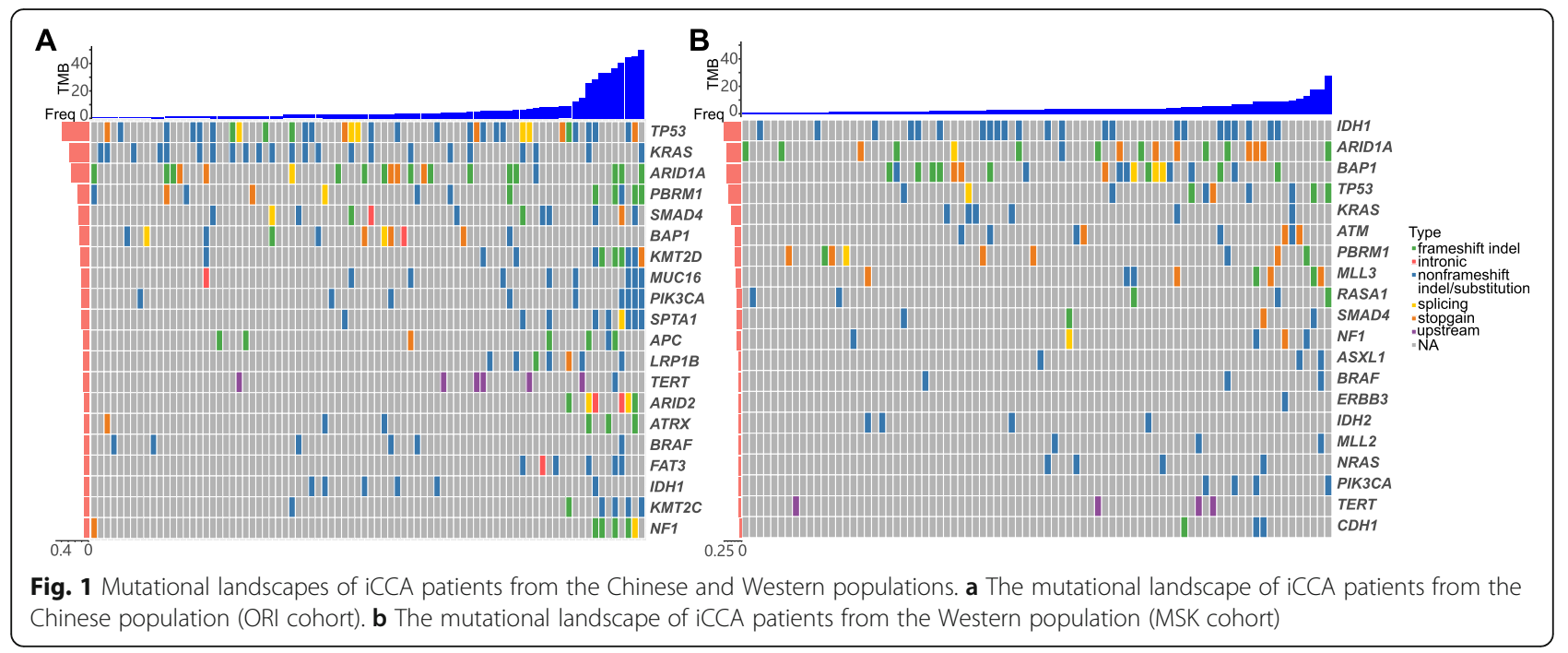

A

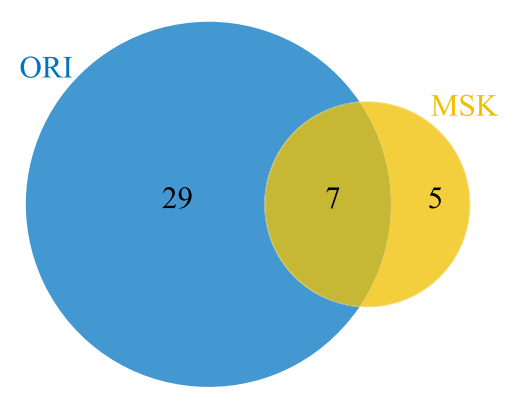

C

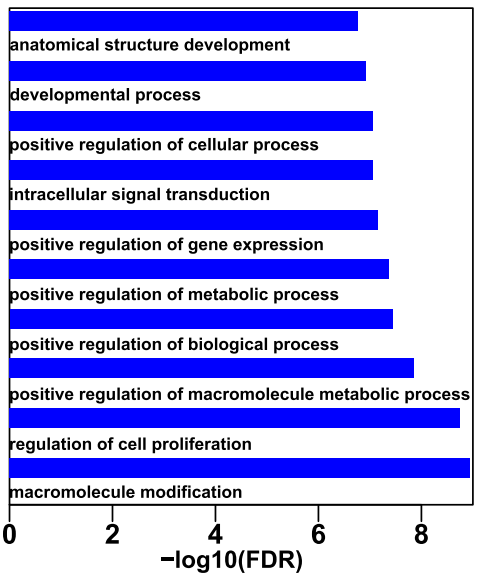

B

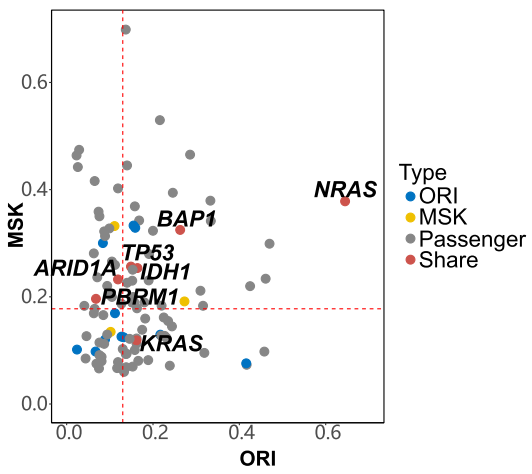

D

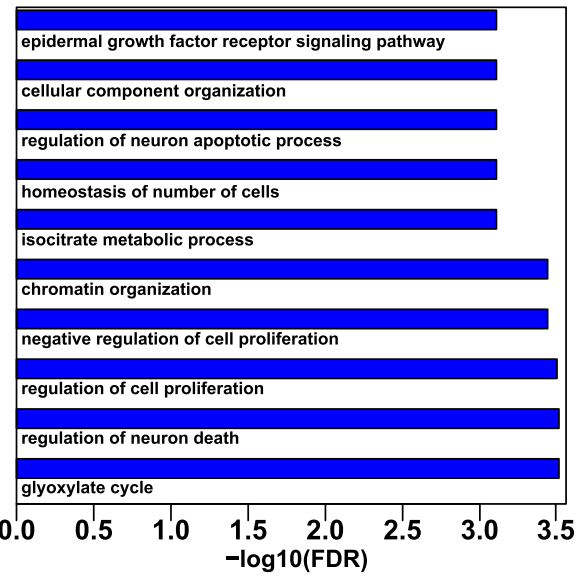

Fig. 2 Significantly mutated genes in both populations. a The significantly mutated gene as revealed by MutSigCV analysis. b The median allele frequency (MAF) of mutations in ORI and MSK cohorts was plotted. The red dashes indicate the median value of MAF in ORI and MSK cohorts. c The biological process of gene ontology was enriched for the ORI cohort. $\mathbf{d}$ The biological process of gene ontology was enriched for the MSK cohort 


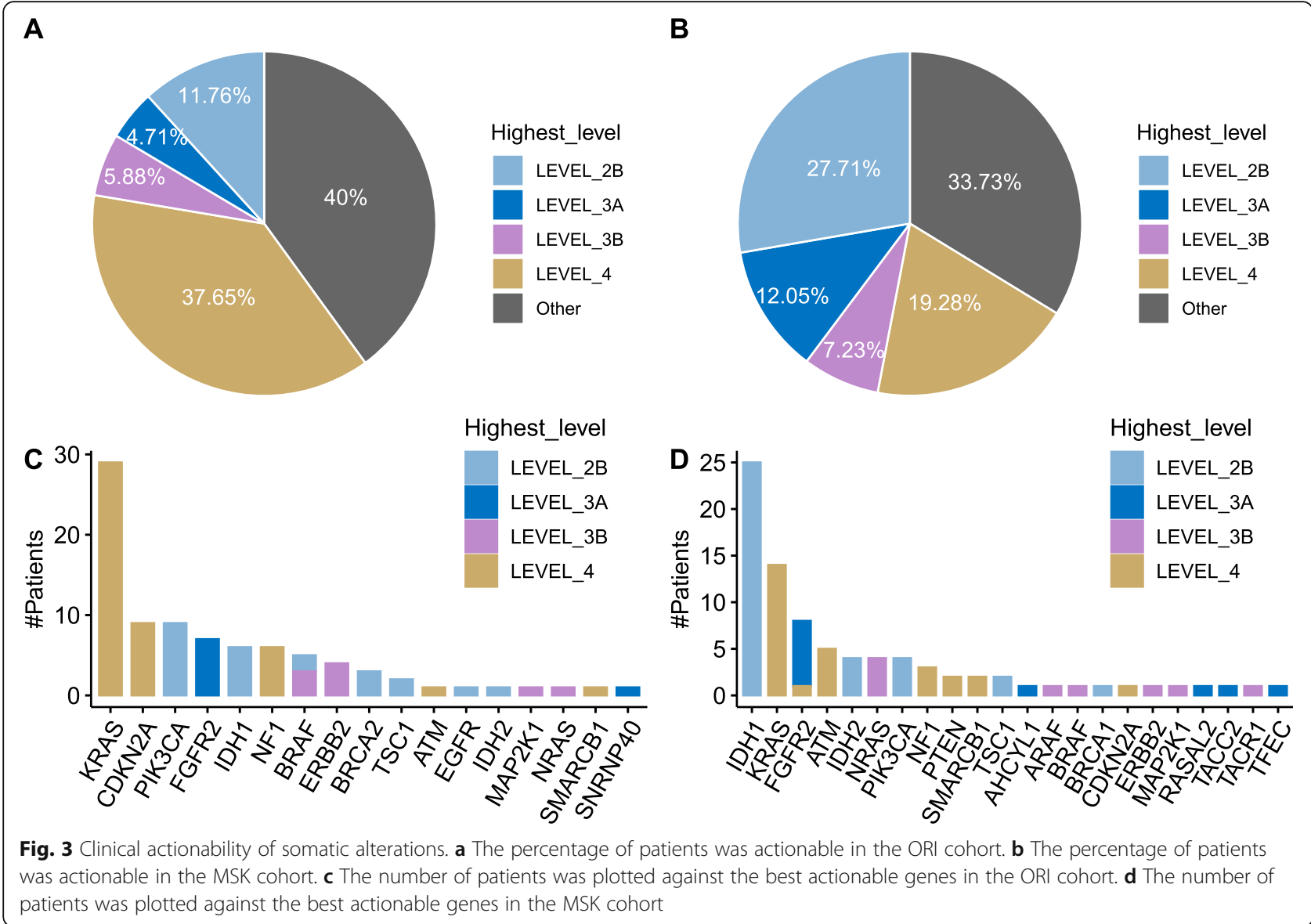

in the MSK cohort only had one mutation (K601E) with Level_3B actionability. For ORI and MSK cohorts, the most actionable mutants were KRAS and $I D H 1$, respectively. Their mutation distribution was depicted in Fig. 4b and Fig. 4c. KRAS had mutations at G12, G13, and Q61 in both cohorts. In the ORI cohort, KRAS also had the other mutations at D57 and D154. IDH1 only had mutations at R132 with Level_2B actionability for both cohorts.

Driver genes correlated with high tumor mutation burden TMB was considered a significant biomarker for immunotherapy efficacy in many other pieces of research. Next, we investigated the TMB distribution in both cohorts. First, the TMB was calculated based on the number of missense mutations across the covered genome length. The median TMB of the ORI cohort was 3.2, ranging from 0.6 to 51.4. The median TMB of the MSK cohort was 2.95, ranging from 0.98 to 27.88 . No significant difference was found between the averages of the two cohorts (Fig. 5a). There were 11 and 5 patients with $\mathrm{TMB}>10 \mathrm{mut} / \mathrm{Mb}$ for the ORI and MSK cohorts, respectively. Among them, two patients without actionable genes had $\mathrm{TMB}>10 \mathrm{mut} / \mathrm{Mb}$ in each cohort. TMB was significantly correlated with KMT2D, MUC16, SPTA1, ARID2, FAT4, FREX2, KMT2C, ACVR2A, LRP1B, and NF1 in the ORI cohort (Fig. 5b-k) and with TP53 and GATA1 in the MSK cohort (Fig. 5, l, m). Among those genes, SPTA1, ARID2, TP53, and GATA1 were found as driver genes in their specific cohorts.

\section{Discussion}

The significantly distinct incidence of iCCA between the Chinese and Western populations had driven us to disclose their genetic and actionable difference. Previously, driver genes were often identified by frequency among the populations. As suggested by Dees et al. [21] and Lawrence et al. [13], high-throughput sequencing studying could bring about many false-positive driver genes because of the mutational heterogeneity on the genome. To circumvent such errors, MutSigCV was employed. MutSigCV analysis had identified 36 significant mutated genes in the ORI cohort. The 10 most significant genes in the ORI dataset were KRAS, TP53, SMAD4, BAP1, IDH1, PBRM1, ARID1A, SPTA1, NTRK3, and STK11, among which NTRK3 and STK11 were not found in the top 20 highly mutated gene list. 


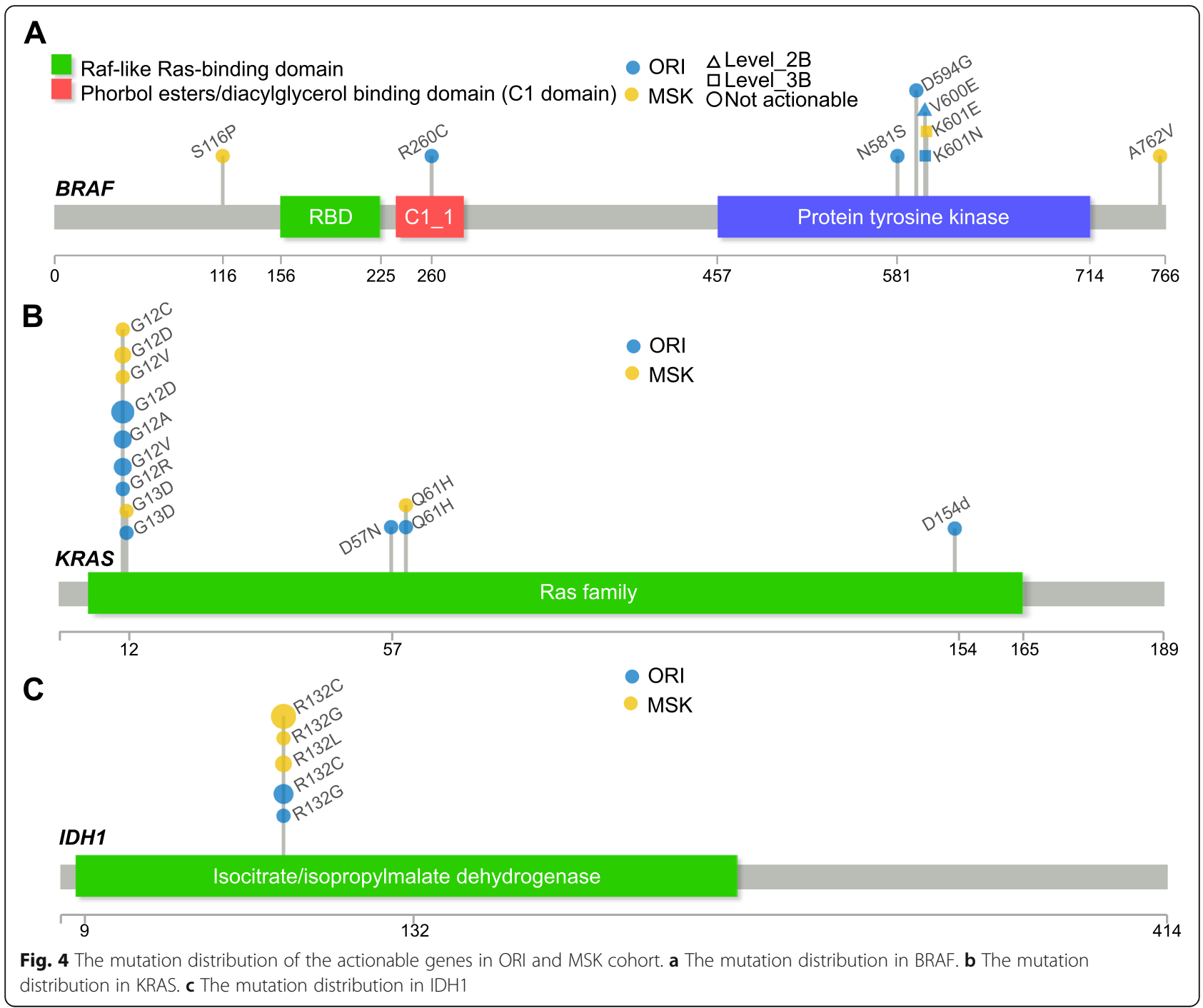

Seven genes (KRAS, TP53, IDH1, ARID1A, PBRM1, $N R A S$, and BAP1) were shared between ORI and MSK cohort. Their mutation prevalence was higher than the median in both cohorts. We compared them to the results from other multiple publications $[3,13]$. The sharing genes included KRAS, TP53, IDH1, and ARID1A, which were driver genes in both ORI and MSK cohorts. The other three genes (PBRM1, NRAS, and BAP1) were not reported in the research of Zou et al. [3] and Lawrence et al. [4]. Actually, NRAS is a very important gene involving many cancer-related signaling pathways, such as MAPK, mTOR, and PI3K-Akt signaling pathways. Currently, NRAS mutation is actionable at level 3B. Patients with NRAS mutation could be treated with Binimetinib or Binimetinib+Ribociclib.

Most iCCA patients can benefit from targeted therapy. About $60 \sim 70 \%$ of patients had actionable mutations in the ORI cohort and MSK cohort. For the actionable mutations, the MSK cohort had a higher actionable level than the ORI cohort, which implied a big potential in the treatment of iCCA for the Chinese population. Of these actionable mutations, KRAS and IDH1 showed a significant bias in actionability between the Chinese and Western populations. The ORI cohort had a higher percentage of KRAS mutation at the actionable level 4 and less percentage of $I D H 1$ mutation at the actionable level 2B. Though KRAS mutations are not targetable directly, patients with KRAS mutation could benefit from inhibition of downstream effectors RAF and MEK. For example, selumetinib (MEK inhibitor) shows a good objective response for metastatic biliary cancer [22] Considering the low evidence proof of KRAS mutations in both populations, drugs targeting downstream of KRAS should be improved urgently. Besides, we also identified fusion events and their actionability. Especially, FGFR2 fusion events most happened in both populations. Targeting FGFR2 fusion could bring about significantly better clinical outcomes. About 33.33 and 

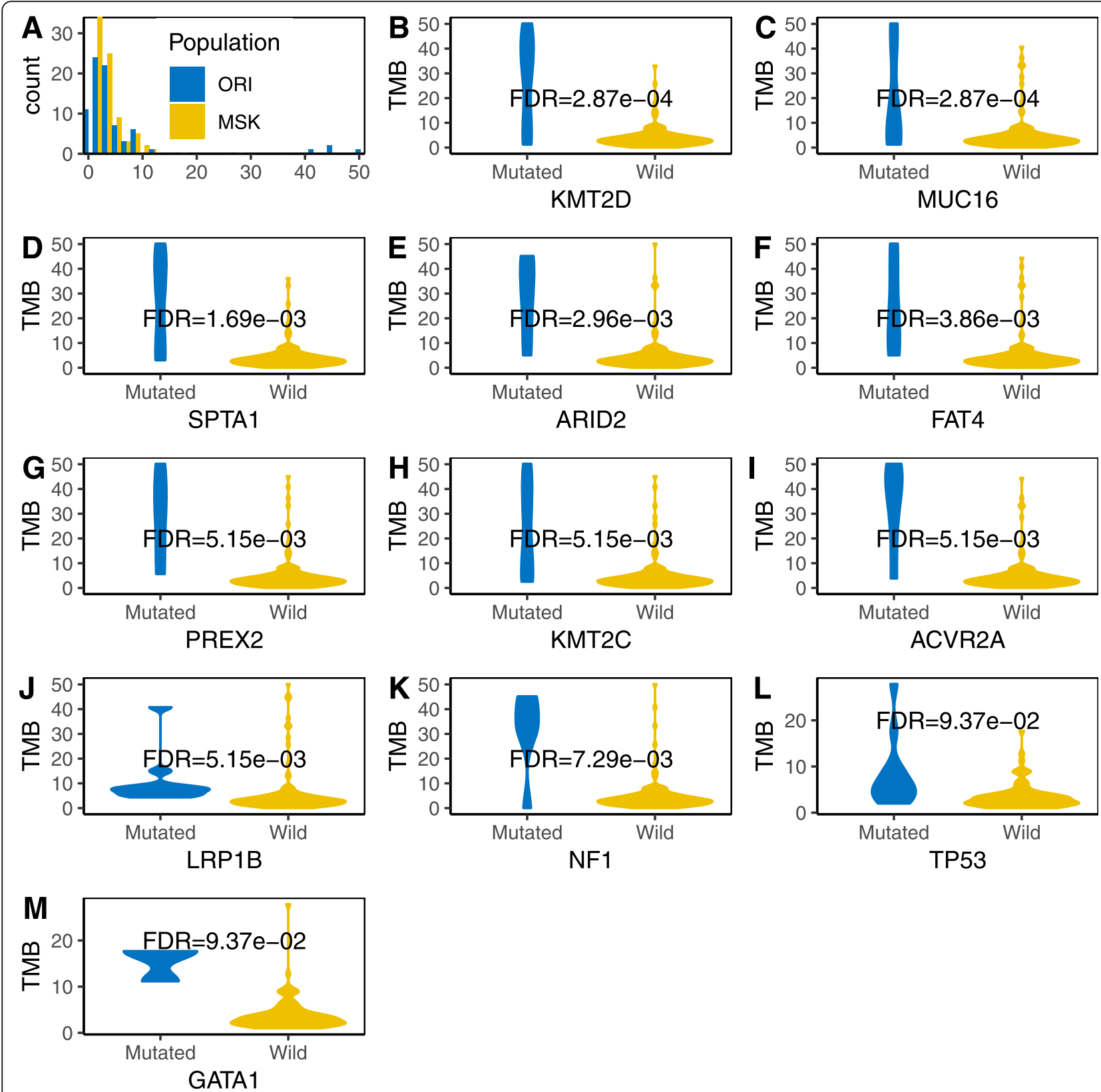

Fig. 5 Genes associated with tumor mutation burden. a The tumor mutation burden (TMB) distribution in both cohorts was plotted. b-k Ten genes were significantly correlated with TMB in the ORI cohort (FDR $<0.01)$. I, $\mathbf{m}$ Ten genes were significantly correlated with TMB in the ORI cohort $(F D R<0.1)$

$57.89 \%$ of patients with FGFR2 fusions could be treated with target therapy at a high actionable level (Level 3A) in the ORI and MSK cohort, respectively. More FGFR2 fusions should be further evaluated for the Chinese population.

Different mutations in the same gene could lead to different optimal treatments. ORI cohort had BRAF mutation V600E at a highly actionable level, 2B. V600E mutation in BRAF was recommended to be treated with Dabrafenib, Dabrafenib+Panitumumab+
Trametinib, Dabrafenib+Trametinib, Encorafenib+ Binimetinib, Encorafenib+Cetuximab+Binimetinib, Trametinib, Vemurafenib, or Vemurafenib+Cobimetinib. MSK cohort had K601E mutation with a lower actionability (level 3B). K601E mutation in BRAF was recommended to be treated with PLX8394.

Immunotherapy also triggered much interest in iCCA treatment. In KEYNOTE-158 (NCT02628067; phase 2) and KEYNOTE-028 (NCT02054806; phase 1b) trials, immunotherapy has shown an improved PFS for 
advanced biliary cancer [23]. Even for refractory unresectable or metastatic biliary cancer, pembrolizumab also exhibited anti-tumor activity [24]. Several case studies also suggested its efficacy. To date, multiple immunotherapy trials are still under study [25]. The Food and Drug Administration even granted accelerated approval to pembrolizumab (KEYTRUDA, Merck \& Co., Inc.) for any unresectable or metastatic tumors with high $\mathrm{TMB}$ $(\mathrm{TMB}>=10 \mathrm{mut} / \mathrm{Mb})$ on June 16,2020 . Though the importance of immunotherapy has been widely accepted, how to improve clinical outcomes is still an urgent issue.

A Mann-Whitney $\mathrm{U}$ test revealed that TMB from both cohorts was not significantly different, which was consistent with the results of previous research [26]. It implied that the higher incidence of iCCA in the Chinese population should not come from a higher mutation rate and immunotherapy might have similar efficacy for both populations. Previously, there were several successful cases of immunotherapy for iCCA. Mou et al. [27] reported a late-stage iCCA Chinese patient with a high TMB. After a combination of anti-PD-1 immunotherapy and chemotherapy, the patient showed a remarkable response. Another Western patient with a high TMB, as reported by Gbolahan et al. [28], gained a good response by taking anti-PD-1 immunotherapy after chemotherapy resistance. Among ORI and MSK cohorts, there were two patients without any actionable mutation but with a higher TMB (> 10 mut/Mb) who could benefit from immunotherapy. Besides, the identified genes that were highly correlated with TMB could be biomarkers of immunotherapy prognosis. For example, MUC16 (CA-125) is a very long protein $(14,500$ amino acids), which is easier to mutate in cancer cells. MUC16 was reported to associate with a higher TMB and a better immunotherapy outcome in gastric cancer [29].

This study aims to investigate the population-wide difference in the landscape of mutations and actionability. The 86 samples from the Chinese population were randomly collected, ignoring the potential subtype preference. The frequency of KRAS and IDHI mutation is similar to the other researches in the coordinate populations [3, 4]. The higher frequency of KRAS mutations in the Chinese population and IDH1 mutations in the Western population could be associated with large duct and small duct iCCA [30, 31], respectively. It is noteworthy that no evidence supports discrimination of small duct from large duct iCCA in targeted therapy though it would be interesting to know whether or not duct types could work as a biomarker of clinical outcomes. We also found that KRAS and IDH1 mutations showed no co-/exclusive occurrence among the ORI and MSK cohorts (Supplemental Fig. 2).

Mutational bias between the two cohorts could be caused by the difference in genetic background, endemic diseases, or special environmental factors. The Chinese population was more affected by liver fluke and hepatic$\mathrm{B}$ virus while the Western population was by primary sclerosing cholangitis and chemical compounds [32-34]. Patients with IDH1 mutation were prone to be flukenegative [35]. But in Western countries, it was still unknown about the risk factor for approximately $50 \%$ of patients [36]. Thus the risk factor of highly mutated IDH1 still needs further investigation. Similarly, KRAS mutations were only found associated with negative HBV without any confirmed risk factor [3]. Another highly mutated gene in the Chinese cohort, TP53, was found associated with liver fluke [35] and hepatic-B virus [3]. In summary, the known risk factors only accounted for a small part of the cases. More efforts are needed to resolve the puzzle.

This study also has its limitations. For example, TMB was calculated from different gene panels for the ORI and MSK cohort, which could weaken the results of the TMB comparison. But according to a more strict comparison, the conclusion still held between the Chinese and Western populations [26]. Besides, because iCCA has a low incidence and patients with it have a short survival time, we failed to enroll more patients to improve the statistical power. Supplemental Table 3 lists the power analysis result of the top 10 mutated genes in the comparison between the two cohorts. To avoid this limitation, we did not directly compare the proportion of each gene between the two cohorts but instead identify the driver genes with MutSigCV [13] and compared them. MutSigCV used patient-specific mutation frequencies and gene-specific background mutation rates, which can largely improve statistical power.

\section{Conclusions}

In summary, this study compared the driver genes, actionability, and TMB in iCCA patients between the Chinese and Western populations. Shared and distinct driver genes were identified. KRAS and IDH1 mutations account for about $20 \% \sim 30 \%$ of actionable targets with a significant bias in the two populations. The drugs targeting KRAS downstream pathway need urgent improvement. Half of the driver genes are still not actionable. The two populations did not show a significant TMB difference.

\section{Supplementary Information}

The online version contains supplementary material available at https://doi. org/10.1186/s12885-021-07792-x.

Additional file 1: Supplemental Table 1. The actionability of driver genes

Additional file 2: Supplemental Table 2. Fusion events 
Additional file 3: Supplemental Table 3. Comparison of mutational frequency for the top 10 mutated genes between the Chinese and Western cohorts.

Additional file 4: Supplemental Figure 1. The enriched KEGG pathways of ORI driver genes

Additional file 5: Supplemental Figure 2. The co-/exclusive occurrence mutations. (A) The co-/exclusive occurrence mutations in the ORI cohort. (B) The co-/exclusive occurrence mutations in the MSK cohort.

\section{Abbreviations}

iCCA: Intrahepatic cholangiocarcinoma; CCA: Cholangiocarcinoma; FDR: False discovery rate; TMB: Tumor mutational burden

\section{Acknowledgments}

The authors would like to thank the anonymous reviewers for the statistical analysis and insightful suggestions and Dr. Leilei Lu for the improvement of the English language.

\section{Authors' contributions}

YJZ and WR designed the study. SX, YG, and YWZ analyzed and interpreted the patient data. SX, YG, XZ, NF, ZZ, GR, YJZ, and WR collected the patient information and samples. SX, YG, YWZ, ZS, XZ, NF, ZZ, GR, YJZ, and WR drafted the manuscript. All authors read and approved the final manuscript.

\section{Funding}

This work was supported by grants from the clinical medicine innovative plan of Jinan (201907073). The funder had no role in the study design, data collection, and analysis, decision to publish, or preparation of the manuscript. None of the authors received a salary from the funder.

\section{Availability of data and materials}

The ORI dataset is not publicly available due to patients' information protection. The MSK dataset is deposited on the cBioportal database (https://www.cbioportal.org/study/summary?id=msk_impact_2017).

\section{Ethics approval and consent to participate}

This study was approved by the ethical committee of the Affiliated Hospital of Qingdao University and Shandong Provincial Hospital Affiliated to Shandong First Medical University. All participants have provided written informed consent for the clinical investigation in this study.

\section{Consent for publication}

Not applicable.

\section{Competing interests}

YWZ and ZJS are employees of Origimed; the other authors declare that they have no competing interests.

\section{Author details}

'Department of Hepatobiliary Surgery, Shandong Provincial Hospital affiliated to Shandong First Medical University, Shandong, China. ${ }^{2}$ Liver Disease Center, The Affiliated Hospital of Qingdao University, Qingdao, China. ${ }^{3}$ Origimed, Shanghai, China. ${ }^{4}$ Organ Transplant Center, The Affiliated Hospital of Qingdao University, Qingdao, China. ${ }^{5}$ Liver Disease Center, The Affiliated Hospital of Qingdao University, Qingdao, China. ${ }^{6}$ Department of Hepatobiliary Surgery, The Fourth Hospital of Hebei Medical University, Hebei, China. 'Oncology Department, The Armed Police Characteristic Medical Center, Hebei, China. ${ }^{8}$ Organ Transplant Center, The Affiliated Hospital of Qingdao University, Haier Road No. 59, Qingdao 266000, Laoshan District, China.

\section{Received: 26 April 2020 Accepted: 5 January 2021}

Published online: 12 February 2021

\section{References}

1. Singal AK, Vauthey JN, Grady JJ, Stroehlein JR. Intra-hepatic cholangiocarcinoma-frequency and demographic patterns: thirty-year data from the M.D. Anderson Cancer center. J Cancer Res Clin Oncol. 2011;137(7):1071-8.

2. Dhanasekaran R, Hemming AW, Zendejas I, George T, Nelson DR, SoldevilaPico C, Firpi RJ, Morelli G, Clark V, Cabrera R. Treatment outcomes and prognostic factors of intrahepatic cholangiocarcinoma. Oncol Rep. 2013; 29(4):1259-67.

3. Zou S, Li J, Zhou H, Frech C, Jiang X, Chu JS, Zhao X, Li Y, Li Q, Wang H, et al. Mutational landscape of intrahepatic cholangiocarcinoma. Nat Commun. 2014:5:5696

4. Lowery MA, Ptashkin R, Jordan E, Berger MF, Zehir A, Capanu M, Kemeny NE, O'Reilly EM, El-Dika I, Jarnagin WR, et al. Comprehensive molecular profiling of intrahepatic and Extrahepatic Cholangiocarcinomas: potential targets for intervention. Clin Cancer Res. 2018;24(17):4154-61.

5. Hogdall D, Larsen OF, Linnemann D, Svenstrup Poulsen T, Hogdall EV. Exome sequencing of 22 genes using tissue from patients with biliary tract cancer. APMIS. 2020;128(1):3-9.

6. Li L, Wang Y, Shi W, Zhu M, Liu Z, Luo N, Zeng Y, He Y. Serial ultra-deep sequencing of circulating tumor DNA reveals the clonal evolution in nonsmall cell lung cancer patients treated with anti-PD1 immunotherapy. Cancer Med. 2019;8(18):7669-78.

7. Liu J, Li G, Guo Y, Fan N, Zang Y. The association between genomic variations and histological grade in hepatocellular carcinoma. Transl Cancer Res. 2020;9(4):2424-33.

8. Li H, Durbin R. Fast and accurate short read alignment with burrowswheeler transform. Bioinformatics. 2009;25(14):1754-60.

9. McKenna A, Hanna M, Banks E, Sivachenko A, Cibulskis K, Kernytsky A, Garimella K, Altshuler D, Gabriel S, Daly M, et al. The genome analysis toolkit: a MapReduce framework for analyzing next-generation DNA sequencing data. Genome Res. 2010;20(9):1297-303.

10. Cibulskis K, Lawrence MS, Carter SL, Sivachenko A, Jaffe D, Sougnez C, Gabriel S, Meyerson M, Lander ES, Getz G. Sensitive detection of somatic point mutations in impure and heterogeneous cancer samples. Nat Biotechnol. 2013;31(3):213-9.

11. Ye K, Schulz MH, Long Q, Apweiler R, Ning Z. Pindel: a pattern growth approach to detect break points of large deletions and medium sized insertions from paired-end short reads. Bioinformatics. 2009;25(21):2865-71.

12. Rausch T, Zichner T, Schlattl A, Stutz AM, Benes V, Korbel JO. DELLY: structural variant discovery by integrated paired-end and split-read analysis. Bioinformatics. 2012;28(18):i333-9.

13. Lawrence MS, Stojanov P, Polak P, Kryukov GV, Cibulskis K, Sivachenko A, Carter SL, Stewart C, Mermel CH, Roberts SA, et al. Mutational heterogeneity in cancer and the search for new cancer-associated genes. Nature. 2013; 499(7457):214-8.

14. Reich M, Liefeld T, Gould J, Lerner J, Tamayo P, Mesirov JP. GenePattern 2.0. Nat Genet. 2006;38(5):500-1.

15. Kanehisa M, Goto S. KEGG: Kyoto encyclopedia of genes and genomes. Nucleic Acids Res. 2000;28(1):27-30.

16. Huang da W, Sherman BT, Lempicki RA. Systematic and integrative analysis of large gene lists using DAVID bioinformatics resources. Nat Protoc. 2009; 4(1):44-57.

17. Chakravarty D, Gao J, Phillips SM, Kundra R, Zhang H, Wang J, Rudolph JE, Yaeger R, Soumerai T, Nissan MH et al. OncoKB: a precision oncology Knowledge Base. JCO Precis Oncol. 2017;Special series(1):1-16.

18. Zehir A, Benayed R, Shah RH, Syed A, Middha S, Kim HR, Srinivasan P, Gao J, Chakravarty D, Devlin SM, et al. Mutational landscape of metastatic cancer revealed from prospective clinical sequencing of 10,000 patients. Nat Med. 2017:23(6):703-13.

19. Gao J, Aksoy BA, Dogrusoz U, Dresdner G, Gross B, Sumer SO, Sun Y, Jacobsen $A$, Sinha $R$, Larsson $E$, et al. Integrative analysis of complex cancer genomics and clinical profiles using the cBioPortal. Sci Signal. 2013;6(269):pl1.

20. Li Cl, Samuels DC, Zhao YY, Shyr Y, Guo Y. Power and sample size calculations for high-throughput sequencing-based experiments. Brief Bioinform. 2018;19(6):1247-55.

21. Dees ND, Zhang Q, Kandoth C, Wendl MC, Schierding W, Koboldt DC, Mooney TB, Callaway MB, Dooling D, Mardis ER, et al. MuSiC: identifying mutational significance in cancer genomes. Genome Res. 2012;22(8):1589-98.

22. Bekaii-Saab T, Phelps MA, Li X, Saji M, Goff L, Kauh JS, O'Neil BH, Balsom S, Balint C, Liersemann R, et al. Multi-institutional phase II study of selumetinib in patients with metastatic biliary cancers. J Clin Oncol. 2011;29(17):2357-63.

23. Piha-Paul SA, Oh DY, Ueno M, Malka D, Chung HC, Nagrial A, Kelley RK, Ros W, Italiano A, Nakagawa K, et al. Efficacy and safety of pembrolizumab for the treatment of advanced biliary cancer: results from the KEYNOTE-158 and KEYNOTE-028 studies. Int J Cancer. 2020.

24. Kang J, Jeong JH, Hwang HS, Lee SS, Park DH, Oh DW, Song TJ, Kim KH, Hwang S, Hwang DW, et al. Efficacy and safety of Pembrolizumab in patients 
with refractory advanced biliary tract Cancer: tumor proportion score as a potential biomarker for response. Cancer Res Treat. 2020;52(2):594-603.

25. Blair $A B$, Murphy A. Immunotherapy as a treatment for biliary tract cancers: a review of approaches with an eye to the future. Curr Probl Cancer. 2018; 42(1):49-58

26. Abdel-Wahab R, Liu S, Cao J, Hu J, Meric-Bernstam F, Xu J, Li Q, Yan M, Feng $Y$, Lin J, et al. AB045. P-13. Genomic heterogeneity between Asian and Western intrahepatic cholangiocarcinoma. Hepatobiliary Surg Nutr. 2019; 8(Suppl 1):AB045.

27. Mou H, Yu L, Liao Q, Hou X, Wu Y, Cui Q, Yan N, Ma R, Wang L, Yao M, et al. Successful response to the combination of immunotherapy and chemotherapy in cholangiocarcinoma with high tumour mutational burden and PD-L1 expression: a case report. BMC Cancer. 2018;18(1):1105.

28. Gbolahan O, Hashemi-Sadraei N, O'Neil B. Prolonged response to anti-PD-1 antibody therapy in chemotherapy-refractory Cholangiocarcinoma with high tumor mutational burden. J Natl Compr Cancer Netw. 2019;17(6):644-8.

29. Li X, Pasche B, Zhang W, Chen K. Association of MUC16 mutation with tumor mutation load and outcomes in patients with gastric Cancer. JAMA Oncol. 2018;4(12):1691-8.

30. Ma B, Meng H, Tian Y, Wang Y, Song T, Zhang T, Wu Q, Cui Y, Li H, Zhang $W$, et al. Distinct clinical and prognostic implication of IDH1/2 mutation and other most frequent mutations in large duct and small duct subtypes of intrahepatic cholangiocarcinoma. BMC Cancer. 2020;20(1):318.

31. Kendall T, Verheij J, Gaudio E, Evert M, Guido M, Goeppert B, Carpino G. Anatomical, histomorphological and molecular classification of cholangiocarcinoma. Liver Int. 2019:39(Suppl 1):7-18

32. Al-Bahrani R, Abuetabh Y, Zeitouni N, Sergi C. Cholangiocarcinoma: risk factors, environmental influences and oncogenesis. Ann Clin Lab Sci. 2013; 43(2):195-210

33. Choi J, Ghoz HM, Peeraphatdit T, Baichoo E, Addissie BD, Harmsen WS, Therneau TM, Olson JE, Chaiteerakij R, Roberts LR. Aspirin use and the risk of cholangiocarcinoma. Hepatology. 2016;64(3):785-96.

34. Clements O, Eliahoo J, Kim JU, Taylor-Robinson SD, Khan SA. Risk factors for intrahepatic and extrahepatic cholangiocarcinoma: a systematic review and meta-analysis. J Hepatol. 2020;72(1):95-103.

35. Jusakul A, Cutcutache I, Yong CH, Lim JQ, Huang MN, Padmanabhan N, Nellore V, Kongpetch S, Ng AWT, Ng LM, et al. Whole-genome and Epigenomic landscapes of etiologically distinct subtypes of Cholangiocarcinoma. Cancer Discov. 2017;7(10):1116-35.

36. Khan SA, Tavolari S, Brandi G. Cholangiocarcinoma: epidemiology and risk factors. Liver Int. 2019;39(Suppl 1):19-31.

\section{Publisher's Note}

Springer Nature remains neutral with regard to jurisdictional claims in published maps and institutional affiliations.

Ready to submit your research? Choose BMC and benefit from:

- fast, convenient online submission

- thorough peer review by experienced researchers in your field

- rapid publication on acceptance

- support for research data, including large and complex data types

- gold Open Access which fosters wider collaboration and increased citations

- maximum visibility for your research: over $100 \mathrm{M}$ website views per year

At BMC, research is always in progress.

Learn more biomedcentral.com/submissions 